This item was submitted to Loughborough's Research Repository by the author.

Items in Figshare are protected by copyright, with all rights reserved, unless otherwise indicated.

\title{
Position tracking control in torque mode for a robotic running foot for footwear testing
}

PLEASE CITE THE PUBLISHED VERSION

https://doi.org/10.1177/1754337118768323

PUBLISHER

SAGE Publications @ IMechE

VERSION

AM (Accepted Manuscript)

\section{PUBLISHER STATEMENT}

This work is made available according to the conditions of the Creative Commons Attribution-NonCommercialNoDerivatives 4.0 International (CC BY-NC-ND 4.0) licence. Full details of this licence are available at: https://creativecommons.org/licenses/by-nc-nd/4.0/

\section{LICENCE}

CC BY-NC-ND 4.0

\section{REPOSITORY RECORD}

Nguyen, Thanh L., Samuel J. Allen, and Soo J. Phee. 2019. "Position Tracking Control in Torque Mode for a Robotic Running Foot for Footwear Testing”. figshare. https://hdl.handle.net/2134/33796. 


\title{
Position tracking control in torque mode for a robotic running foot for footwear testing
}

\author{
NGUYEN Thanh Luan ${ }^{1,2,3}$, Samuel James ALLEN ${ }^{3}$, and PHEE Soo Jay, Louis ${ }^{2}$ \\ 1 School of Mechanical \& Aerospace Engineering, Nanyang Technological University, Singapore \\ 2 Institute for Sports Research, Nanyang Technological University, Singapore \\ 3 School of Sport, Exercise and Health Sciences, Loughborough University, UK
}

\begin{abstract}
Available automatic footwear testing systems still lack flexibility and bio-fidelity to represent the human foot and reproduce the wear conditions accurately. The first part of this paper introduces a new design of the robotic running foot (RRF) for footwear testing using cable conduit mechanisms (CCMs). This RRF is integrated with an upper leg mechanism to form a complete integrated footwear testing system (IFTS). The CCMs help remove the bulky actuators and transmissions out of the fast-moving robotic foot. Thus, this RRF design not only allows high-power actuators to be installed, but also avoids a significant dynamic mass and inertia effects on the upper leg mechanism. This means that the IFTS can have multiple powered degrees of freedom (DOFs) in the RRF and simulate much higher human running speeds than other available systems. However, CCMs cause significant challenges in control approaches, especially in high-speed systems, due to their nonlinear transmission characteristics. Furthermore, the RRF actuators must operate in a torque/force control mode to reproduce the foot-shoe interaction during gaits while it is critical to control the foot joints position in the swing phase of gaits. The latter part of this paper presents a study on position tracking control in torque mode for the RRF joints using adaptive and PID (Proportional-Integral-Derivative) control designs to evaluate the system's ability to mimic the human foot kinematics in running. Both controllers proved their effectiveness, implying that the proposed control approach can be implemented on the IFTS to control the foot joints' position in the swing phase of running gaits.
\end{abstract}

\section{Introduction}

A new footwear design can be tested by using mechanical devices, computer simulation methods, or some human wear participation trials. The human participation method involves a group of wearers using the new shoe model for a period of several months, after which the shoe mechanical properties are compared[11, 13, 12]. The human participation method is time-consuming and very costly with a limited number of participating subjects and testing trials. Moreover, this approach is not suitable for certain tests, such as shock absorption ability, which can expose the participants to the injury risks. Meanwhile, the computer simulation methods (e.g., finite element methods), which face significant challenges in modeling test conditions as well as the complex human foot geometry and anatomy, can be inaccurate and unrealistic[4]. Hence, the approach of using mechanical devices, which overcomes these limitations and offers other advantages (e.g., stable repeatability, adjustable condition parameters, etc.), is preferred in footwear testing.

The prior art of footwear testing devices has witnessed numerous machines[1, 28, 14, 6, 25, 24, 5, 29, 27, 3] which were able to replicate different wear conditions (e.g., the impact, friction, abrasion with the ground, humidity, and temperature of the environment) to evaluate the materials and design of the shoes. Nevertheless, these devices were not able to realistically replicate the interaction between the foot and the shoe in running because they all employed fixed prosthetic feet with no active degree of freedom (DOF) for wearing the testing shoe. This paper introduces a new design of the robotic running foot (RRF) for footwear testing[20, 22, 21] which has potentials to mimic human running gaits.

Furthermore, when a robotic system simulates a human gait, the system must cope with the discrete loading condition during a gait cycle. In running gaits, a cycle is usually defined as it begins when a foot contacts the ground and ends when the same foot contacts the ground again. A running cycle includes two primary phases: the contact phase when a foot is in contact with the ground, and the swing phase when that foot is in the air. In the contact phase, the foot endures a very large ground reaction force (GRF), which is up to three times the runners body mass $[23,26]$. However, in the swing phase, the load is significantly lower than the GRF in the contact phase. The swing load includes only the total foot-shoe inertia and the friction of the foot joints at the running speeds. Thus, this discrete loading condition, which occurs at a very high speed during the running cycle (i.e., less than one second per cycle[23]), causes a great challenge for any robotic system to mimic/replicate the running gaits.

During the contact phase, the robotic foot must mimic the GRF profile of the human gait and thus, the actuators should operate in a torque/force control mode. On the other hand, the RRF must mimic the correct angles of the human foot joints to replicate the right effects on the shoe when the foot-shoe lands and impacts with the ground. Thus, the control strategy is to alternate the control target between position control in the swing phase and torque/force control in the contact phase. This paper addresses the position control in torque mode for the robotic foot joints which are actuated by the cable conduit mechanisms (CCMs). 


\section{New Design of the Robotic Foot for Footwear Testing}

The basic ideas of the RRF for footwear testing were introduced in the authors' previous papers[20, 22, 21]. The robotic foot consists of two powered joints at the ankle and metatarsophalangeal (MTP) joints. Each joint is actuated by a pair of CCMs. The actuating principles of this robotic foot and the structure of an upper leg mechanism were described in Nguyen et al.[22, 21]. A proposed structure of the integrated footwear testing system (IFTS) and the fundamental of a new RRF design are illustrated in Fig. 1. Compared to the previous design[22], the current RRF includes an additional timing belt transmission for actuating the ankle joint and uses a 3D-printed foot tissue instead of a carbon fiber leaf spring for absorbing the impact shock. More specifically, while the Achilles Tendon (AT) cable and Tibialis Anterior (TA) cable control the ankle joint, they do not connect directly to the foot (i.e., the ankle pulley). These cables connect to an auxiliary pulley (i.e., the ankle distal pulley), which is fixed to the smaller pulley of the timing belt transmission. This timing belt mechanism helps amplify the active torque for the ankle joint. In human running gaits, the ankle active torque is extremely high (e.g., up to $3.44 \mathrm{Nm}$ per $\mathrm{kg}$ of the runner's body mass[26]). Thus, this timing belt transmission allows the system to power the ankle joint up to the required torque while maintaining a lower tension and friction on the CCMs. Meanwhile, the Toe Flexor (TFL) cable and Toe Extensor (TEX) cable actuate the MTP joint. Each pair of CCMs are controlled by a powerful actuator which is mounted on a stationary control table. The structure of these two actuators of the RRF is also illustrated in Fig. 1. Each actuator comprises a powerful 3-phase servomotor, a low backlash gear reduction box, a high-accuracy torque sensor which is supported by two high-stiffness zero-backlash couplings, a proximal pulley of the CCMs, and a proximal incremental encoder.

More details of the transmission principle of a pair of CCMs are described in Fig. 2. The proximal pulley actuates the robotic joint by pulling Cable 1 or Cable 2, while two ends of each conduit are fixed to the robot frame. The length of the conduit is considered as constant when the cable is pulled. This makes the motion/force be propagated from the proximal pulley to the robotic joint. Specifically, the clockwise torque/motion from the motor side (i.e., proximal pulley) is propagated to the robot side (i.e., distal pulley) by pulling Cable 1, while the two ends of Conduit 1 are fixed. On the contrary, when the proximal pulley rotates counter-clockwise, it pulls Cable 2 and drives the robotic joint rotating counter-clockwise. More details on the actuating mechanisms and actuators of the RRF joints were described in Nguyen et al.[22]. Figure 1 also shows a proposed fundamental structure for the upper-leg mechanism. It consists of three other powered DOFs (i.e., the translational motions 1 to 3 ). The first two translational actuators drive the foot-shank block horizontally and rotate it in the sagittal plane following the human foot profile. The third actuator elevates the footshank block and applies the body mass effects to the foot.

The CAD model of the new RRF design is portrayed in Fig. 3 while its current actual fabricated model is shown in Fig. 4. The current RRF includes three primary seg- ments: toes, foot, and lower shank. These segments connect at the ankle and MTP joints. On each joint, a highresolution encoder is installed to monitor its angle feedback for the closed-loop control. A Scancon 2RMHF-7500 incremental encoder with 7500 pulses/revolution is used for the ankle joint, while the MTP joint is supported by a smaller Scancon SCH-16F-5000 encoder with a resolution of 5000 pulses/revolution. In this current RRF design, the carbon fiber leaf spring is obsolete and a 3D-printed foot model is developed to cover the foot and toes main segments. This 3D-printed foot (subsequently referred to as the 'foot-tissue') has been developed based on a CT (computerized axial tomography) scan foot model[18] (size US 9 ). The foot-tissue is divided into three parts for assembling around the foot and toes main structure. In Fig. 3 , these parts are rendered transparently to display the inside main structure and the MTP encoder. The outer shape of these 3D-printed parts can be observed in Fig. 4. The CT-scan foot model copies a static human foot model and thus, the 3D-printed foot has the shape of a static human foot. The main purpose of those powered foot joints is to mimic the effects of the foot dynamic shape during gaits. The foot-tissue was printed using ABSplus ${ }^{\mathrm{TM}}$ thermoplastic material by the Fortus 250mc 3D-printer from Stratasys Ltd.

\section{Position Tracking Control in Torque Mode at Human Run- ning Speeds}

The RRF is designed to mimic both kinematic and kinetic behaviors of the human foot, especially the interaction force/pressure between the foot and the shoe. During each gait cycle, it is crucial to replicate correctly this interaction force/pressure in the contact phase because it directly influences the deformation and durability of the footwear. Subsequently, in the swing phase of a gait cycle, the foot position must be reset to the right position when that foot starts to contact with the ground to simulate correctly the impact effects during landing. As discussed in the Introduction, the proposed control strategy is to alternate the control targets between position control and torque control in the two phases of a running cycle, i.e., the swing phase and contact phase, respectively. Consequently, the controller and servo drives must operate in a torque control mode in which the primary control signal is a torque signal but not a common voltage signal. The second control signal is a maximum speed signal which is used to prevent the motor from exceeding a certain safety velocity when the external loading torque suddenly drops. Both torque control and limit speed signals are required to control the output torque and motion of the servomotors and actuators of the RRF joints.

This paper focuses on mimicking the ankle angle profile in human running gaits since the MTP joint has a smaller range of motion and relatively slower speeds. More specifically, with the position feedback at the ankle joint and ankle proximal pulley, two closed-loop control schemes were adopted and implemented in real-time experiments. A simple traditional PID controller and a nonlinear adaptive controller were developed to compare their performance. 


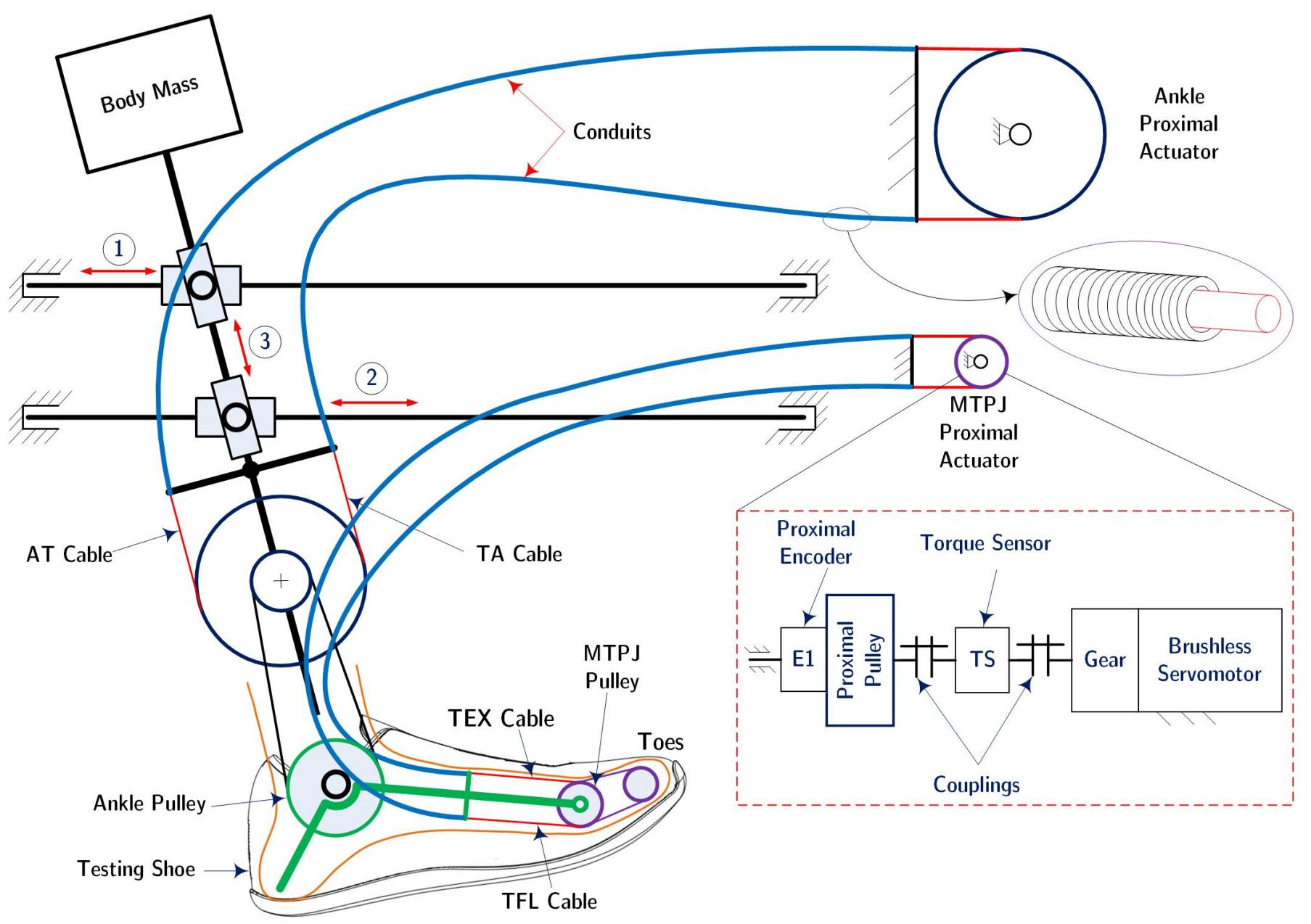

Figure 1: Schematic Structure of the Robotic Running Foot (RRF) in the Integrated Footwear Testing System (IFTS). The RRF includes two active joints. The ankle joint is powered by the ankle pair of cable conduit mechanisms (CCMs): Achilles Tendon (AT) cable and Tibialis Anterior (TA) cable. The metatasophalangeal (MTP) joint is actuated by the MTP pair of CCMs: Toe Flexor (TFL) cable and Toe Extensor (TEX) cable.

The chosen reference signal was a sinusoidal signal with a frequency of $7.27 \mathrm{rad} / \mathrm{s}(\approx 1.157 \mathrm{~Hz})$ and a range of motion of $50^{\circ}$. This reference is equivalent to an ankle angle profile in normal running gaits at about $12-14 \mathrm{~km} / \mathrm{h}$ (i.e., a gait cycle period is 0.864 second and an ankle angle ranges from $25^{\circ}$ dorsi-flexion to $25^{\circ}$ plantar-flexion[23, 26]). The following sections present the methods, experiments, and results of recent position tracking control in torque mode to this reference profile $\varphi_{r}(t)=25^{\circ} \sin (1.157 \times 2 \pi t)$.

\subsection{Problem Formulation}

From Fig. 2, one can observe the following dynamic equation at the distal pulley of the pair of CCMs as shown in Eq. (1):

$$
\tau_{d}=J_{d} \ddot{\varphi}_{d}+\nu_{d} \dot{\varphi}_{d}+\tau_{o}
$$

in which $\tau_{d}$ is the distal pulley active torque, $\tau_{o}$ is the output torque at the distal pulley; $J_{d}$ and $\nu_{d}$ represent the inertia and viscous friction at the distal pulley; $\dot{\varphi}_{d}$ and $\ddot{\varphi}_{d}$ are the distal pulley angular velocity and acceleration; the dot and double-dot on top of variables represent their first and second derivatives with respect to time. Without losing generality, this paper focuses on the ankle joint, which includes an additional timing belt transmission between the actuator and the ankle joint. The formulation for the
MTP joint is relatively simpler since its distal pulley is installed directly on the joint. Let $J$ and $\nu$ be the equivalent total inertia and friction factors of the ankle distal pulley, the foot-shoe block, and the timing belt transmission at the ankle axis, so that one can obtain the following Eq. (2) at the ankle joint:

$$
\tau_{d}=J \ddot{\varphi}+\nu \dot{\varphi}+\tau_{l}
$$

in which $\tau_{l}$ is the output loading torque at the ankle joint. From the characteristics of a pair of CCMs, one can write a balance torque equation of two pulleys as shown in Eq. (3):

$$
\tau_{p}=\tau_{d}+T_{\text {fric }}
$$

in which $\tau_{p}$ is the active torque at the proximal pulley. The friction on a pair of CCMs can be modeled as in Do et al.[8] by using a Bouc-Wen hysteresis model[15] as shown in Eqs. (4) and (5):

$$
\begin{aligned}
T_{\text {fric }} & =\kappa_{\varphi} \varphi_{p}+\kappa_{\eta} \eta+\nu_{c} \dot{\varphi}_{p}+T_{0} \\
\dot{\eta} & =\rho\left[\dot{\varphi}_{p}-\sigma\left|\dot{\varphi}_{p}\right||\eta|^{n-1}+(\sigma-1) \dot{\varphi}_{p}|\eta|^{n}\right]
\end{aligned}
$$

in which $\rho, \sigma$, and $n$ are scalar parameters that control the shapes and size of the hysteresis loop; $\kappa_{\varphi}$ and $\kappa_{\eta}$ represent the scale factors of the friction to the input displacement 


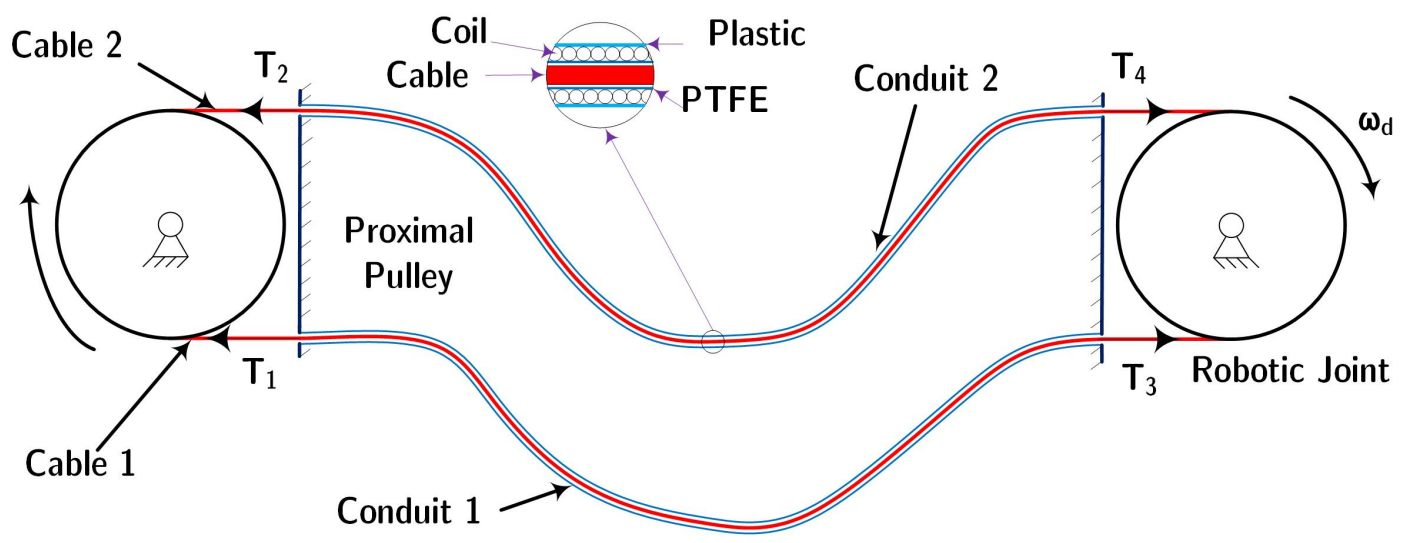

Figure 2: The transmission fundamental of a pair of cable-conduit mechanisms (CCMs).

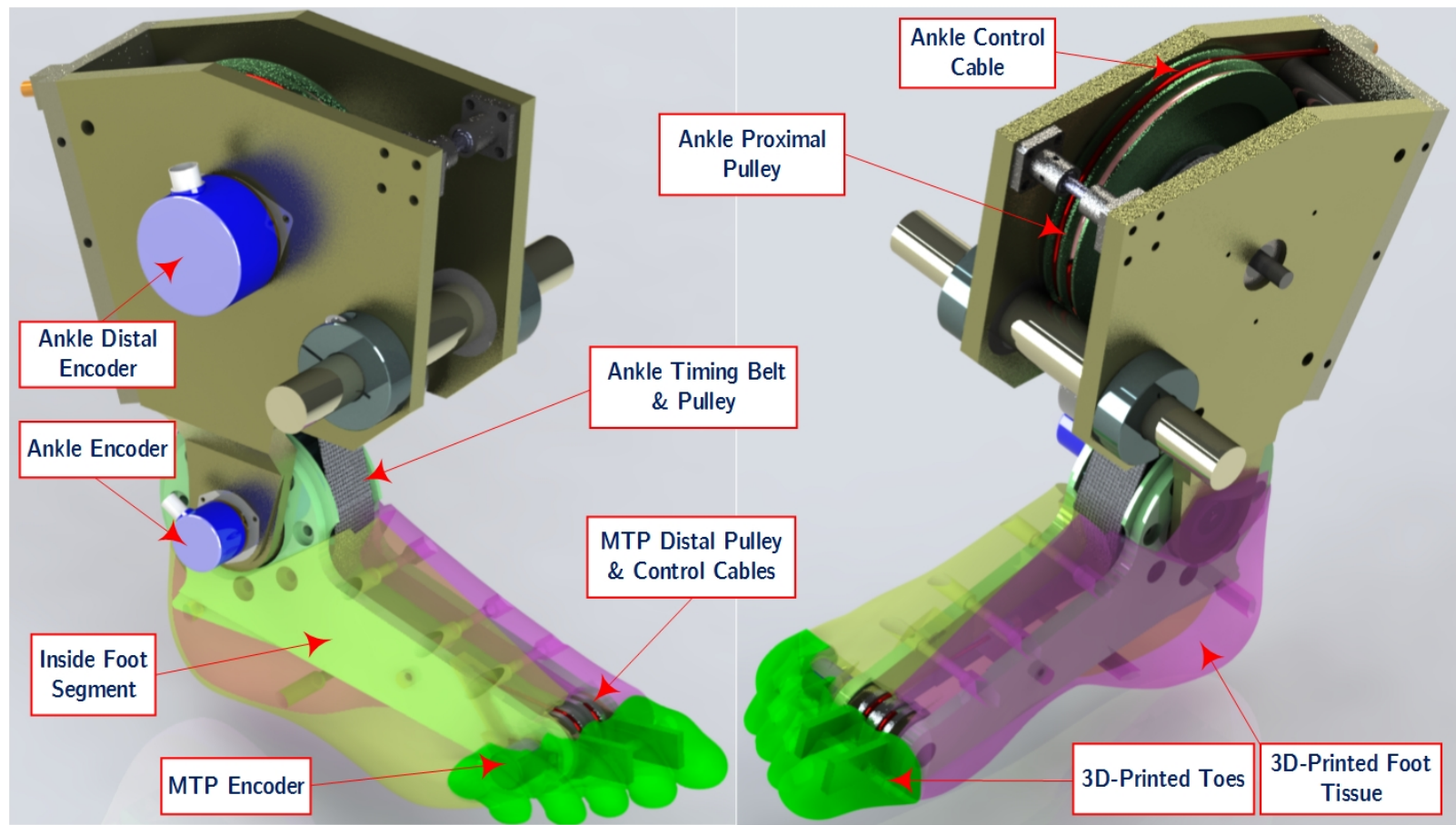

Figure 3: The CAD model of the new Robotic Running Foot (RRF) for Footwear Testing

$\varphi_{p}$ and the internal state $\eta$ respectively; $\dot{\varphi}_{p}$ is the relative velocity at the proximal pulley. Furthermore, if one denotes $J_{p}$ and $\nu_{p}$ as the total inertia and viscous friction factors on the proximal pulley and gearbox of the actuator, the dynamic equation at the proximal side is shown in Eq. (6)

$$
A u_{c}=J_{p} \ddot{\varphi}_{p}+\nu_{p} \dot{\varphi}_{p}+\tau_{p}
$$

in which $\ddot{\varphi}_{p}$ is the angular acceleration of the proximal pulley; $u_{c}$ is the torque control signal and $A$ is a positive coefficient. The term $A u_{c}$ represents an approximation of the actuator output torque $\tau_{m}$. This approximation was obtained based on the guaranteed effectiveness of the high-speed torque control loop of the actuator servo drive. Hence, from Eqs. (2) - (6), one can obtain Eqs. (7):

$$
\begin{aligned}
A u_{c}=J_{p} \ddot{\varphi}_{p}+\left(\nu_{p}+\nu_{c}\right) \dot{\varphi}_{p}+\kappa_{\varphi} \varphi_{p}+\kappa_{\eta} \eta+T_{0} \\
+J \ddot{\varphi}+\nu \dot{\varphi}+\tau_{l} .
\end{aligned}
$$

This paper focuses on motion control in the swing phase of running. The target is to push quickly and smoothly the foot joints to the right positions to prepare for landing with correct effects. Thus, there is no GRF or $\tau_{l}=0$ in this case. Denote $\varphi_{r}(t)$ as the reference ankle angle profile that the actuator must control the ankle joint to track to. Let $\varphi(t)$ be the feedback output of the ankle angle, then the tracking error is as shown in Eq. (8):

$$
e(t)=\varphi(t)-\varphi_{r}(t) .
$$

Furthermore, the following assumptions are made to design an appropriate controller for the robotic foot joints:

(i) Sufficient pretension is applied to the pair of CCMs to prevent any cable slacking.

(ii) The reference profile $\varphi_{r}(t)$ and its first two timederivatives $\dot{\varphi}_{r}(t)$ and $\ddot{\varphi}_{r}(t)$ are explicit and bounded.

(iii) The output position feedback $\varphi(t)$ and its first timederivatives $\dot{\varphi}(t)$ are bounded. 


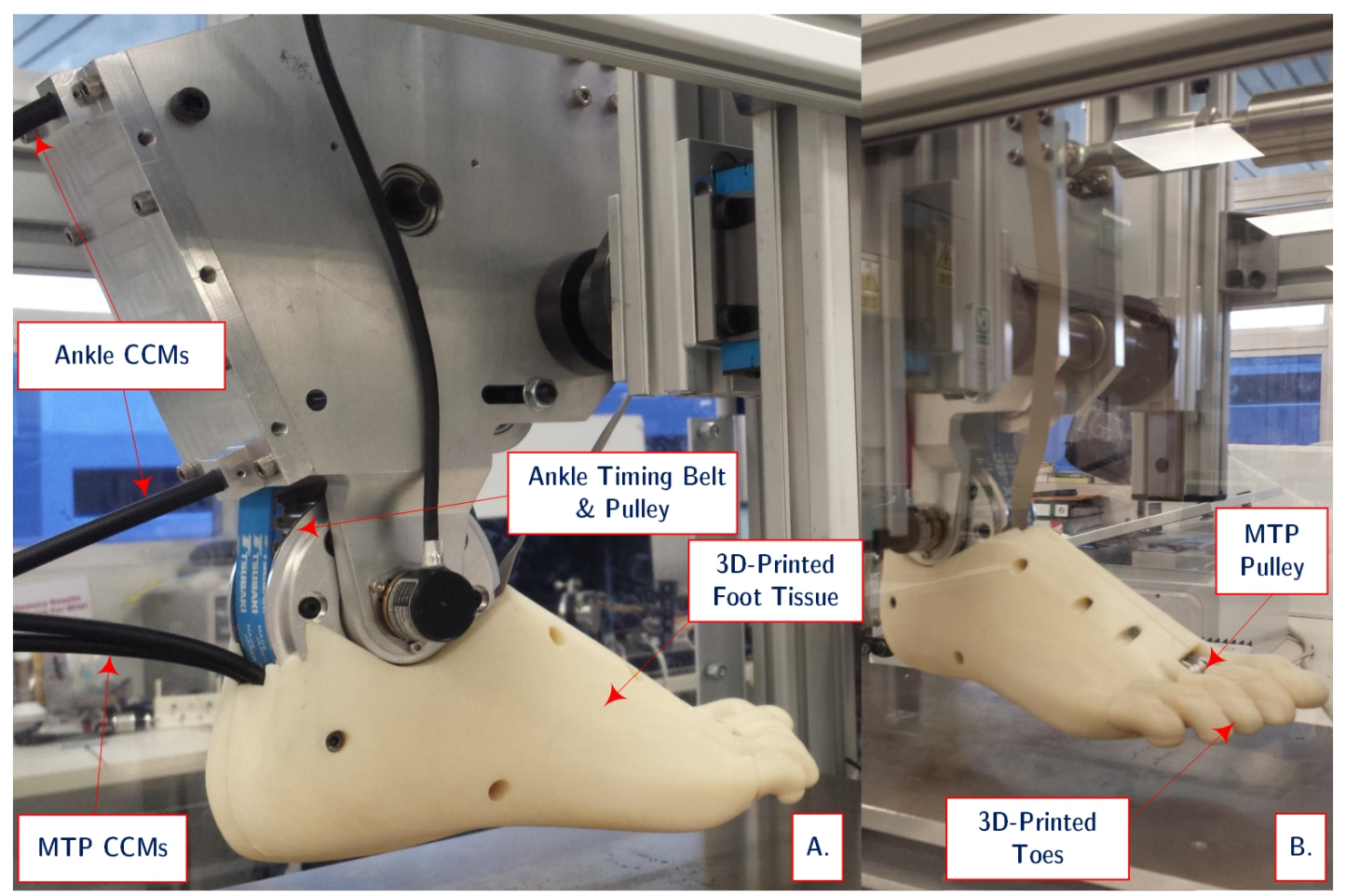

Figure 4: The real Robotic Running Foot (RRF) in the Integrated Footwear Testing System (IFTS)

\subsection{PID Control Design}

In this control scheme, the torque control signal can be calculated as follows in Eq. (9):

$$
u_{c}=-K e(t)-K_{I} \int_{0}^{t} e(\omega) d \omega-K_{D} \frac{d e(t)}{d t}
$$

in which $K, K_{I}$, and $K_{D}$ are positive control gains. In addition, in this torque mode control, the servomotor speed is saturated by a maximum speed derived by as shown in Eq. (10):

$$
u_{V}(t)=\left|k_{V} e(t)+k_{V I} \int_{0}^{t} e(\omega) d \omega\right|
$$

\subsection{Robust Adaptive Control Design}

To develop an adaptive control scheme, another assumption is made:

(iv) The first two time-derivatives of the proximal pulley angle, which is measured by the proximal encoder, are assumed bounded.

The system torque-balance Eq. (7) can be rewritten as shown in Eq. (11):

$$
\left\{\begin{array}{l}
\dot{x}_{1}=x_{2} \\
\dot{x}_{2}=\alpha u_{c}-\xi^{T} \theta-h-b x_{2} \\
y=\varphi=x_{1}
\end{array}\right.
$$

in which $x_{1}=\varphi$ and $x_{2}=\dot{x}_{1}=\dot{\varphi}$ represent the output state variables; $\alpha=A / J, b=\nu / J, \theta_{1}=\kappa_{\varphi} / J$, $\theta_{2}=\left(\nu_{p}+\nu_{c}\right) / J, \theta_{3}=J_{p} / J$ are all positive parameters; $\xi^{T}=\left(\xi_{1}, \xi_{2}, \xi_{3}\right)$ is the transpose vector of the secondary state variables: $\xi_{1}=\varphi_{p}, \xi_{2}=\dot{\varphi}_{p}$, and $\xi_{3}=\ddot{\varphi}_{p}$; and $\theta=\left(\theta_{1}, \theta_{2}, \theta_{3}\right)^{T}$ is a parameter vector formed by above $\theta_{i}(i=1,2,3)$. Also, $h=\left(\kappa_{\eta} \eta+T_{0}\right) / J$ represents the total hysteresis and offset friction on the pair of CCMs. This unknown parameter includes the effects of the variable transmission route, external disturbance, and pretension condition on the pair of CCMs.

To design an adaptive control law for this system, one can define new filter tracking errors $z_{1}$ and $z_{2}$ as follows in Eq. (12):

$$
\left\{\begin{array}{l}
z_{1}=x_{1}-\varphi_{r} \\
z_{2}=\dot{z}_{1}+\lambda z_{1}
\end{array}\right.
$$

in which $\lambda$ is an arbitrary positive coefficient. Then they yield Eq. (13):

$$
\left\{\begin{aligned}
\dot{z}_{1} & =x_{2}-\dot{\varphi}_{r}=z_{2}-\lambda z_{1} \\
\dot{z}_{2} & =\dot{x}_{2}-\ddot{\varphi}_{r}+\lambda \dot{z}_{1} \\
& =\alpha u_{c}-\xi^{T} \theta-h-b x_{2}-\ddot{\varphi}_{r}+\lambda z_{2}-\lambda^{2} z_{1}
\end{aligned}\right.
$$

A new virtual control signals $\bar{u}_{c}$ is introduced to derive the control signal $u_{c}$ as $u_{c}=\widehat{\psi} \bar{u}_{c}$, where $\widehat{\psi}$ is an estimate of the new control gain $\psi=1 / \alpha$. Let $\widetilde{\psi}$ be the estimated error of $\psi$ as $\widetilde{\psi}=\psi-\widehat{\psi}$. From above definitions, the control term in Eq. (13) can be expressed in terms of the new virtual control signal $\bar{u}_{c}$ as follows in Eq. (14):

$$
\alpha u_{c}=\alpha \widehat{\psi} \bar{u}_{c}=\alpha(\psi-\widetilde{\psi}) \bar{u}_{c}=\bar{u}_{c}-\alpha \widetilde{\psi} \bar{u}_{c}
$$

Denote $H>0$ as the bound of the total hysteresis and offset friction on the pair of CCMs $h(t)$ (i.e., $|h| \leq H$ ); and denote $\widehat{\theta}_{i}, \hat{b}$, and $\hat{H}$ as the estimates of parameters $\theta_{i}$, $b$, and $H$; their corresponding estimated errors are $\widetilde{\theta}_{i}=$ $\theta_{i}-\widehat{\theta}_{i}, \tilde{b}=b-\hat{b}$, and $\tilde{H}=H-\hat{H}$. Then, the control and update laws can be designed as follows in Eqs. (15) and 
(16):

$$
\begin{gathered}
\left\{\begin{array}{l}
u_{c}=\widehat{\psi} \bar{u}_{c} \\
\bar{u}_{c}=-(k+\lambda) z_{2}+\left(\lambda^{2}-1\right) z_{1}+\ddot{\varphi}_{r} \\
+\hat{b} x_{2}+\sum_{i=1}^{3} \widehat{\theta}_{i} \xi_{i}-\hat{H} \operatorname{sign}\left(z_{2}\right)
\end{array}\right. \\
\begin{cases}\dot{\hat{\psi}}=-\delta z_{2} \bar{u}_{c}-\sigma_{\psi} \widehat{\psi} \\
\hat{\hat{b}}=-\varrho z_{2} x_{2}-\sigma_{b} \hat{b} \\
\dot{\hat{H}}=\mu\left|z_{2}\right|-\sigma_{H} \hat{H} \\
\dot{\dot{\theta}_{i}}=-\gamma_{i} z_{2} \xi_{i}-\sigma_{i} \widehat{\theta}_{i}\end{cases}
\end{gathered}
$$

in which positive parameters $\gamma_{i}, \varrho, \delta$, and $\mu$ are the estimating gains, positive coefficients $k$ and $\lambda$ represent the control gains. In addition, the leakage terms $-\sigma_{\psi} \widehat{\psi},-\sigma_{b} \hat{b}$, $-\sigma_{H} \hat{H}$, and $-\sigma_{i} \widehat{\theta}_{i}$ are added to the parameter estimating laws in Eq. (16) to prevent parameter drifting phenomena[16]. The speed of the servomotor is still controlled by the maximum speed as in Eq. (10).

Theorem 1. Consider the dynamic system in Eq. (11) which satisfies Assumptions (i) - (iv), with the control and update laws Eqs. (15) and (16), the following statements hold.

a. All signals of the resulting closed-loop system are uniformly ultimately bounded.

$b$. The tracking errors $z_{1}$ and $z_{2}$ converge to a neighborhood of zero with the bound defined by $\Delta=\sqrt{2 \Phi / v}$, in which Eq. (17) applies:

$$
\left\{\begin{aligned}
v & =\min \left(2 \lambda, 2 k, \sigma_{\psi}, \sigma_{i}, \sigma_{b}, \sigma_{H}\right) \\
\Phi & =\sigma_{\psi} \alpha \psi^{2} / 2 \delta+\sum_{i=1}^{3}\left(\sigma_{i} \theta_{i}^{2} / 2 \gamma_{i}\right) \\
& +\sigma_{b} b^{2} / 2 \varrho+\sigma_{H} H^{2} / 2 \mu
\end{aligned}\right.
$$

\subsection{Experiments and Results}

These two closed-loop control designs have been implemented in Matlab-Simulink and loaded into a real-time controller (dSPACE DS1104) to compare their performance. This real-time controller can monitor angle feedback from encoders of the proximal pulley and ankle joint. In the PID control scheme, only ankle joint kinematics are used to compute the control signal, while the robust adaptive control scheme requires signals of both proximal and output sides.

Upon receiving the feedback angles, the controller executes the control law to drive the ankle joint to track to the same reference profile $\varphi_{r}(t)=25^{\circ} \sin (1.157 \times 2 \pi t)$. The controller DS1104 monitors the output feedback $\varphi(t)$ and proximal position feedback $\varphi_{p}(t)$ and follows either Eqs. (9) or (15) to compute a torque control signal $u_{c}$. Table 1 shows design values for the control and estimating gains in the control and update laws in Eqs. (15) and (16) of the robust adaptive control scheme. Firstly, $\sigma_{i}, \sigma_{\psi}, \sigma_{b}$ and $\sigma_{H}$ in the $\sigma$-modification leakage terms are selected at a reasonably small value to minimize the residue errors due to the leakage modifications. They were set to have $1 \%$ of the corresponding estimated parameter values. Secondly, the estimating gains $\gamma_{i}, \delta, \varrho$, and $\mu$ were set at very small values. Meanwhile, $k$ and $\lambda$ were increased slowly until the output position tracked to the reference. Finally, these estimating gains $\gamma_{i}, \delta, \varrho$, and $\mu$ were increased slowly to accelerate the parameters estimation process and improve the output tracking performance. This table also lists the initial estimate of the model parameters. Simultaneously, DS1104 also calculates the limit speed signal $u_{V}$ from the formula in Eq. (10). After that, the central controller DS1104 sends these two control signals to the servo drive M701-03400100A (Control Technique, Emerson, US) to control the servomotor 115U2D300 (Control Technique) of the ankle actuator (as illustrated in Fig. 1). These signals are sent through two 16-bit digital-to-analog conversion (DAC) modules.

During a control trial, the configuration of the pair of CCMs was randomly changed due to the movement of the robotic foot. It is noted that the two control designs do not require the knowledge of the CCM configuration. The adaptive control scheme will estimate online the transmission characteristics and calculate an appropriate control signal to compensate for the nonlinear hysteresis, external disturbance, and unknown configuration. On the other hand, the PID control scheme simply linearizes the transmission of the whole model and uses only the output feedback to compute its control signal with a proportional gain $K$, an integral gain $K_{I}$, and a derivative gain $K_{D}$. Table 2 shows the designed values of these PID control gains. They are designed by online manual tuning with the system and real-time feedback. Firstly, $K_{I}$ was set to zero while $K_{D}$ was set to a very small value compared to $K$. Then, $K$ was increased slowly until the measured output tracked closely to the reference position and then the output started oscillating around the reference profile. Then, $K_{I}$ was increased slowly until any offset between reference and feedback was reduced significantly. During this process, $K_{D}$ could be increased (slower than $K_{I}$ ) to reduce overshoot and oscillation.

Figure 5 plots the tracking data at the stable states of the PID (upper panel) and robust adaptive (lower panel) control schemes in driving the ankle joint following above sinusoidal reference profile. The root mean square error (RMSE) of nine control trials for each control scheme is shown in Table 3. Both control schemes performed effectively. The RMSE of the PID control trials was $0.8001^{\circ} \pm 0.0051^{\circ}$ which is approximately $1.60 \%$ of the reference range. This is slightly smaller than the RMSE of adaptive control trials $\left(0.8109^{\circ} \pm 0.0305^{\circ}\right)$. Furthermore, the transient performance of the two schemes in the same control trials is plotted in Fig. 6. Both controllers also performed well at the initial states. It is noted that the current robust adaptive controller is slightly more sensitive and less stable than the PID controller.

Hence, the PID controller was chosen to implement on both ankle and MTP joints to control their position in mimicking human running gaits. As illustrated in Fig. 1 , both actuators of the foot joints have the same principle structure including a servomotor, gear reduction box, torque sensor supported by two couplings, proximal pulley driving two CCMs, and an incremental encoder attached to the proximal pulley. The same control approach of the ankle joint is applied to the MTP joint. Figure 7 plots the tracking performance of the PID control scheme in driving these two joints tracking to real reference data of a running trial at $12 \mathrm{~km} / \mathrm{h}[19]$. Tracking errors to these real human running data were quite stable with RMSE for the ankle and MTP joints of $1.1437^{\circ}$ and $0.9702^{\circ}$, respectively. 
In addition, on the specific periods of contact phases, the RMSEs between the references and feedbacks were $1.2725^{\circ}$ for the MTP joint $\left(\approx 4.02 \%\right.$ of the joint range) and $1.2536^{\circ}$ for the Ankle joint $(\approx 2.29 \%$ of the joint range).

Table 1: Design parameters for the robust adaptive controller

Adaptive Gains
$k$
$\lambda$
$\delta$
$\mu$
$\rho$
$\gamma_{1}$
$\gamma_{2}$
$\gamma_{3}$

\begin{tabular}{c||cc} 
Values & Parameters & Initial Values \\
300 & - & - \\
3.5 & - & - \\
0.0001 & $\hat{\psi}_{0}$ & 0.01 \\
0.4 & $\hat{H}_{0}$ & 200 \\
0.05 & $\hat{b}_{0}$ & 0 \\
0.1 & $\hat{\gamma}_{1} 0$ & 0 \\
0.05 & $\hat{\gamma}_{2} 0$ & 0 \\
0.01 & $\hat{\gamma}_{3} 0$ & 0
\end{tabular}

Table 2: Design parameters for the PID controller

\begin{tabular}{c|ccc} 
PID Gains & Proportional $K$ & Integral $K_{I}$ & Derivative $K_{D}$ \\
Values & 18 & 3 & 0.432
\end{tabular}

\section{Discussion}

\subsection{The new robotic running foot for footwear testing}

The new RRF is a unique artificial foot with multiple powered DOFs for footwear testing. To the authors' understanding, the available footwear testing systems[1, 28, 14, $6,25,24,5,29,27,3]$ use only fixed prosthetic feet or imitative foot lasts to wear the testing shoe. Thus, with the ability to mimic more movements of the human foot, the new RRF is expected to replicate more accurately the interaction force/pressure between the human foot and the shoe. This would help footwear developers evaluate their design more accurately. The 3D-printed foot-tissue also duplicates a real human foot model which can result in more realistic interaction pressure with the testing shoe.

The new RRF design has potentials because of the use of CCMs to power the foot joints. If a robotic foot or prosthesis employs traditional transmission mechanisms such as gears, belts, timing belts, or ball-screw transmissions, the upper leg mechanism must bear all of their bulky components. Then, the more power the foot joints require, the more cumbersome these transmissions and actuators must be. Notably, in human running gaits, the ankle joint conveys a significant amount of power and moment[23, 26]. As a result, these joints' actuator and transmission mechanisms cause an enormous dynamic mass and inertia to the upper leg mechanism in the fast movement of running gaits. This requires a more powerful upper leg mechanism. Furthermore, when this robotic foot impacts the ground to simulate human running gaits, all 'intelligent' and delicate components (e.g., sensors and control circuits), which are attached to it, would be damaged rapidly. These are some reasons that available footwear testing systems do not have powered foot joints and cannot simulate human running gaits properly to test the shoe.

On the other hand, the flexible transmission route and ability to remotely place the bulky actuator from the out-
Table 3: The root mean square error (RMSE) of nine typical control trials of two control schemes.

\begin{tabular}{c|c|c} 
Control Trials & PID Control & Robust Adaptive Control \\
1 & $0.8010^{\circ}$ & $0.8055^{\circ}$ \\
2 & $0.8026^{\circ}$ & $0.8117^{\circ}$ \\
3 & $0.7944^{\circ}$ & $0.8430^{\circ}$ \\
4 & $0.8068^{\circ}$ & $0.7801^{\circ}$ \\
5 & $0.8011^{\circ}$ & $0.8466^{\circ}$ \\
6 & $0.8044^{\circ}$ & $0.7720^{\circ}$ \\
7 & $0.8000^{\circ}$ & $0.8431^{\circ}$ \\
8 & $0.8018^{\circ}$ & $0.7651^{\circ}$ \\
9 & $0.7888^{\circ}$ & $0.8314^{\circ}$ \\
Average & $0.8001^{\circ}$ & $0.8109^{\circ}$ \\
$\%$ to Max Range & $1.6002 \%$ & $1.6219 \%^{\circ}$ \\
SD & $0.0051^{\circ}$ & $0.0305^{\circ}$
\end{tabular}

put joint allow the CCMs to actuate the RRF effectively. The CCMs are very suitable for applications which require high payload, compact design, and small weight and inertia such as for rehabilitation[31], exoskeletons[30, 17], and anklefoot prosthesis[2]. With CCMs, all motors, gearboxes, and strain gauge sensors can be removed from the robotic foot, while the control torques/motions are propagated through the CCMs. Thus, CCMs not only enable the possibility of increasing the joint power, but also eliminate the high inertial effects caused by bulky motors and actuators on the fast-moving RRF. Also, removing the actuators and transmissions from the RRF frees space for adding other structures, strengthens the imitative foot, and makes it more realistic. This method also avoids the risks of damaging other electronic components and sensors because they are placed at the proximal side of the CCMs. In addition, the cable of CCMs can be considered as a high stiffness spring and can replace the elastic component of the series elastic actuators. These represent great advantages of the CCMs in developing a robotic foot for footwear testing. However, CCMs have a limited useful life when conveying large forces and operating continuously in a long period of footwear testing. Thus, the users may need to maintain and replace new sets of CCMs more often than other transmission types.

\subsection{Motion control in torque mode for the RRF joints}

The importance of replicating the foot-shoe interaction force/pressure require the controller/actuator of the RRF for footwear testing to operate in a torque control mode. This means that the prime control signal is a direct torque signal, but not a voltage signal (for regulating the servomotor output velocity). Thus, both control targets (i.e., torque output target in the contact phase and position output target in the swing phase of running) must be controlled in this torque mode. Based on the designed structure of the RRF, a dynamic torque-balance as shown in Eq. (7) was established. This model characterizes nonlinear friction and hysteresis of CCMs by using Bouc-Wen hysteresis model[15, 8]. CCMs nonlinear friction and hysteresis caused significant error in control performance and can be compensated for by adaptive control laws[9, 7, 22]. Hence, an elaborate robust adaptive controller and a traditional PID controller are designed and evaluated to compare their performance in position control in torque mode 

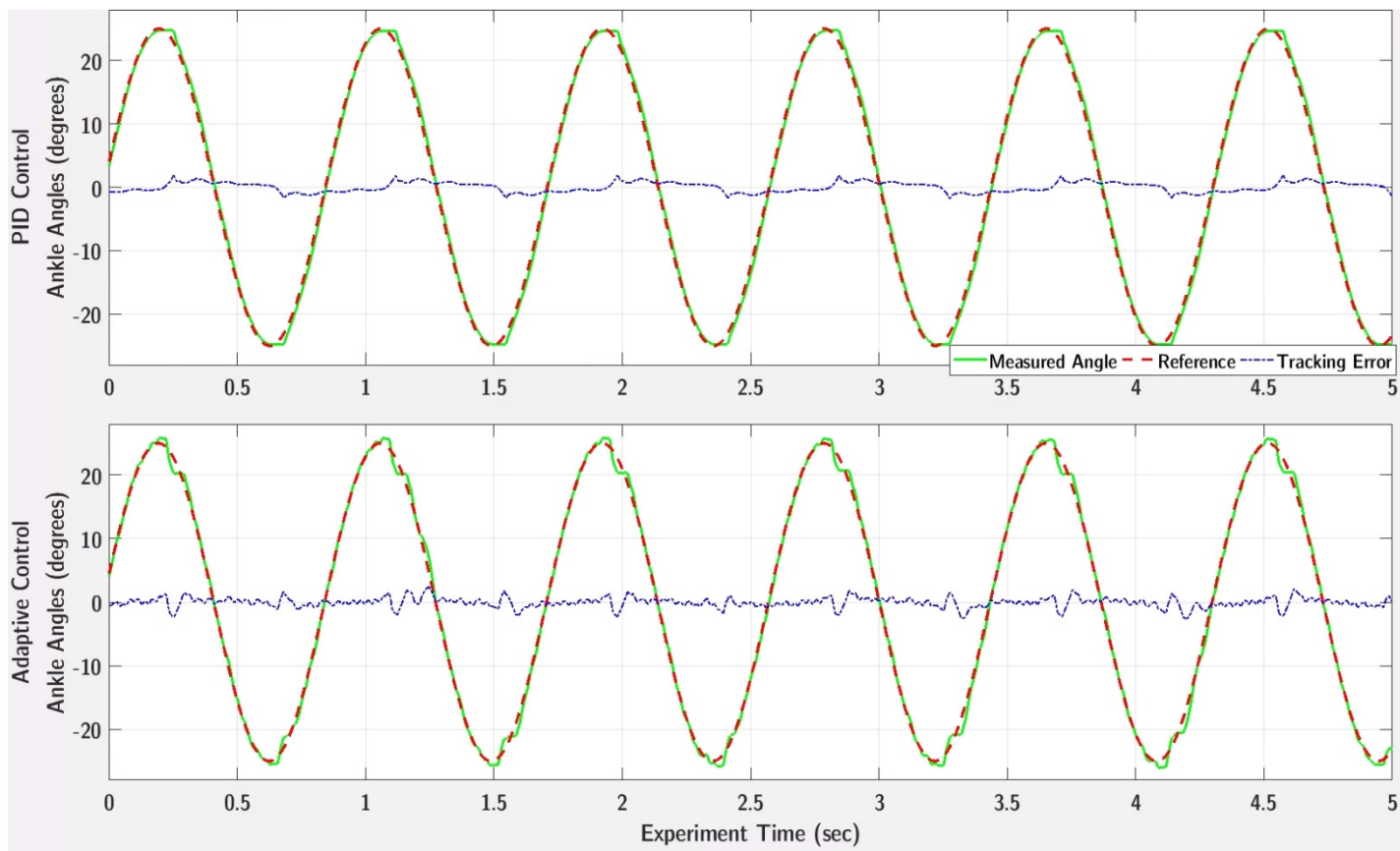

Figure 5: The position tracking performance of the PID and Robust Adaptive Control schemes (at stable states) to the same sinusoidal reference signal $\varphi_{r}(t)=25^{\circ} \sin (1.157 \times 2 \pi t)$ which has the same frequency and range of motion to an ankle angle profile during heelstrike running gaits[23, 26].

for the RRF joints.

The adaptive control is expected to perform better since it takes into account the effects of nonlinear hysteresis, dynamic friction and inertia and uses online estimation to compensate for these effects. However, this adaptive control design contains the first and second time-derivatives of the feedback output and proximal pulley angles which are measured by two incremental encoders. These encoders provide finite discretizing output. Their outputs are also affected by external vibration. As a result, their derivatives more likely contain steep pikes and discontinuity. These effects make the control performance more sensitive to external disturbance. Consequently, a simple traditional PID controller proves that it is slightly more effective than the adaptive controller. The average RMSEs of two control schemes are very close $\left(0.8001^{\circ}\right.$ versus $\left.0.8109^{\circ}\right)$, but the standard deviation of adaptive control RMSEs is considerably larger. Its tracking error graphs show more small fluctuations as well. However, overall, both controllers prove their potential in position tracking performance in torque mode for the RRF joints at human running speeds. Their RMSEs are approximately $1.6 \%$ of the range of motion. In common control applications, the required accuracy ranges from $2 \%$ to $5 \%$. Also, both controllers perform very well at the initial state of the control trials. Notably, with its simpler design and effectiveness, the PID control is more suitable for implementing on the RRF in the IFTS. The results in Fig. 7 show that the designed PID control scheme can provide good performance in position tracking to real human running profiles of the ankle and MTP joints.

\section{Conclusion}

The contemporary footwear testing devices which use conventional actuators and fixed prosthetic feet are not able to replicate properly the dynamic effects on the footwear, and thus do not evaluate the footwear under the most realistic testing conditions. With the use of CCMs, this current RRF can possess multiple powered DOFs to simulate more human foot movements, avoid bulkiness and high dynamic inertia effects, and has ability to mimic human running gaits.

Furthermore, experimental results of position tracking control for the foot joints demonstrate the capability of the RRF in replicating the human foot kinematics in running when the system works in the torque control mode. This could lead to a high possibility of success in the next experiments on replicating both control targets in different phases of human running gaits (i.e., to control output torque/force during the contact phase and output position during the swing phase).

Thus, future work may include designing and implementing control algorithms and experiments on smoothly switching between torque reference control and position reference control in this high-speed system. In addition, complete integration with the upper leg mechanism and footwear testing experiments could be conducted.

\section{A Proof of Theorem 1}

A Lyapunov function can be defined as shown in Eq. (18):

$$
V=\frac{z_{1}^{2}}{2}+\frac{z_{2}^{2}}{2}+\frac{\alpha \widetilde{\psi}^{2}}{2 \delta}+\frac{\tilde{b}^{2}}{2 \varrho}+\sum_{i=1}^{3} \frac{\widetilde{\theta}_{i}^{2}}{2 \gamma_{i}}+\frac{\tilde{H}^{2}}{2 \mu}
$$




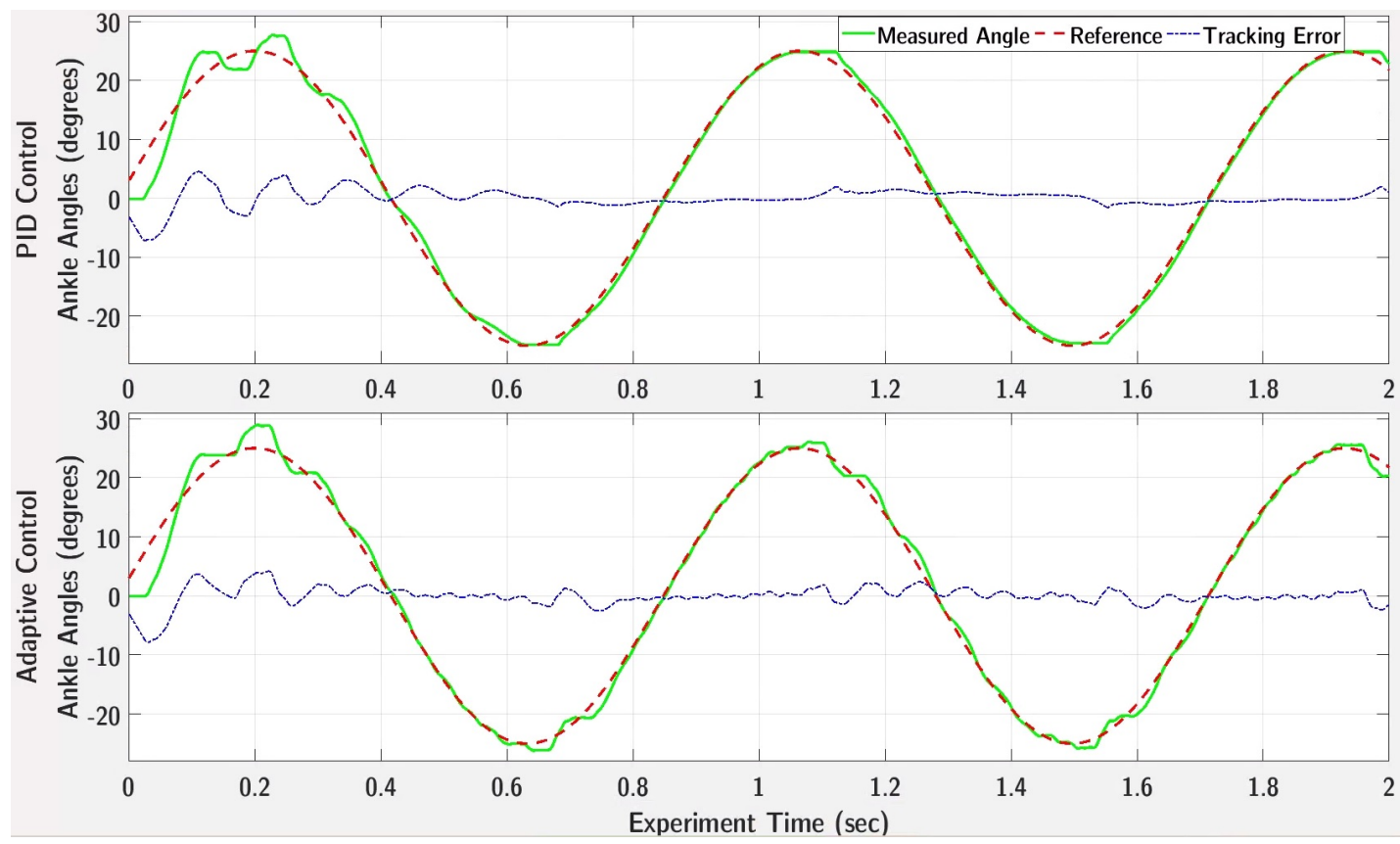

Figure 6: The transient tracking performance of the PID and Robust Adaptive Control schemes to the same sinusoidal reference signal $\varphi_{r}(t)=25^{\circ} \sin (1.157 \times 2 \pi t)$ which has the same frequency and range of motion to an ankle angle profile during heelstrike running gaits[23, 26].

Then its first time-derivative is as shown in Eq. (19):

$$
\begin{aligned}
\dot{V} & =z_{1} \dot{z}_{1}+z_{2} \dot{z}_{2}+\frac{\alpha \tilde{\psi} \dot{\widetilde{\psi}}}{\delta}+\sum_{i=1}^{3} \frac{\widetilde{\theta}_{i} \dot{\tilde{\theta}}_{i}}{\gamma_{i}}+\frac{\tilde{b} \dot{\tilde{b}}}{\varrho}+\frac{\tilde{H} \dot{\tilde{H}}}{\mu} \\
& =-\lambda z_{1}^{2}+z_{1} z_{2}-\frac{\alpha \widetilde{\psi} \dot{\hat{\psi}}}{\delta}-\sum_{i=1}^{3} \frac{\widetilde{\theta}_{i} \dot{\hat{\theta}}_{i}}{\gamma_{i}}-\frac{\tilde{b} \hat{\hat{b}}}{\varrho}-\frac{\tilde{H} \dot{\hat{H}}}{\mu} \\
& +z_{2}\left(\alpha u_{c}-\xi^{T} \theta-h-b x_{2}-\ddot{\varphi}_{r}+\lambda z_{2}-\lambda^{2} z_{1}\right)
\end{aligned}
$$

Substituting Eq. (14) into the last step produces Eq. (20):

$$
\begin{aligned}
\dot{V} & =-\lambda z_{1}^{2}+z_{1} z_{2}-\widetilde{\psi}\left(\frac{\alpha \dot{\hat{\psi}}}{\delta}+\alpha \bar{u}_{c} z_{2}\right)-\frac{\tilde{H} \dot{\hat{H}}}{\mu} \\
& -\sum_{i=1}^{3} \widetilde{\theta}_{i}\left(\frac{\dot{\hat{\theta}}_{i}}{\gamma_{i}}+\xi_{i} z_{2}\right)-\tilde{b}\left(\frac{\dot{\hat{b}}}{\varrho}+x_{1} z_{2}\right)-h z_{2} \\
& +z_{2}\left(\bar{u}_{c}-\sum_{i=1}^{3} \widehat{\theta}_{i} \xi_{i}-\hat{b} x_{2}-\ddot{\varphi}_{r}+\lambda z_{2}-\lambda^{2} z_{1}\right)
\end{aligned}
$$

As $h \leq H$ and $-h z_{2} \leq H\left|z_{2}\right|$, one can obtain Eq. (21):

$$
\begin{aligned}
\dot{V} & \leq-\lambda z_{1}^{2}-\widetilde{\psi}\left(\frac{\alpha \dot{\hat{\psi}}}{\delta}+\alpha \bar{u}_{c} z_{2}\right)-\frac{\tilde{H} \dot{\hat{H}}}{\mu} \\
& -\sum_{i=1}^{3} \widetilde{\theta}_{i}\left(\frac{\dot{\hat{\theta}}_{i}}{\gamma_{i}}+\xi_{i} z_{2}\right)-\tilde{b}\left(\frac{\dot{\hat{b}}}{\varrho}+x_{2} z_{2}\right) \\
& +(\hat{H}+\tilde{H})\left|z_{2}\right|+z_{2}\left(\bar{u}_{c}-\sum_{i=1}^{3} \widehat{\theta}_{i} \xi_{i}\right) \\
& +z_{2}\left(-\hat{b} x_{2}-\ddot{\varphi}_{r}+\lambda z_{2}+\left(1-\lambda^{2}\right) z_{1}\right)
\end{aligned}
$$

Substituting control and update laws Eqs. (15) and (16) into above inequality yields Eq. (22):

$$
\begin{aligned}
\dot{V} & \leq-\lambda z_{1}^{2}-k z_{2}^{2}+\frac{\alpha \sigma_{\psi}}{\delta} \widehat{\psi} \widetilde{\psi} \\
& +\sum_{i=1}^{3} \frac{\sigma_{i}}{\gamma_{i}} \widehat{\theta}_{i} \widetilde{\theta}_{i}+\frac{\sigma_{b}}{\varrho} \hat{b} \tilde{b}+\frac{\sigma_{H}}{\mu} \hat{H} \tilde{H}
\end{aligned}
$$

On the other hand, the last term in Eq. (22) can be rewritten as shown in Eq. (23):

$$
\begin{aligned}
\frac{\sigma_{H} \hat{H} \tilde{H}}{\mu} & =\frac{\sigma_{H}}{\mu} \tilde{H}(H-\tilde{H})=\frac{\sigma_{H}}{\mu}\left(H \tilde{H}-\tilde{H}^{2}\right) \\
& =\frac{\sigma_{H}}{\mu}\left[\frac{H^{2}}{2}-\frac{H^{2}-2 H \tilde{H}+\tilde{H}^{2}}{2}-\frac{\tilde{H}^{2}}{2}\right] \\
& =\frac{\sigma_{H}}{\mu}\left[-\frac{\tilde{H}^{2}}{2}-\frac{1}{2}(\tilde{H}-H)^{2}+\frac{H^{2}}{2}\right] \\
& \leq-\sigma_{H} \frac{\tilde{H}^{2}}{2 \mu}+\frac{\sigma_{H} H^{2}}{2 \mu}
\end{aligned}
$$

Similarly, other leakage terms can be expressed as follows in Eqs. (24 - 26):

$$
\begin{aligned}
& \frac{\sigma_{b}}{\varrho} \hat{b} \tilde{b} \leq-\sigma_{b} \frac{\tilde{b}^{2}}{2 \varrho}+\frac{\sigma_{b} b^{2}}{2 \varrho} \\
& \frac{\alpha \sigma_{\psi}}{\delta} \widehat{\psi} \widetilde{\psi} \leq-\sigma_{\psi} \frac{\alpha \widetilde{\psi}^{2}}{2 \delta}+\frac{\sigma_{\psi} \alpha \psi^{2}}{2 \delta} \\
& \sum_{i=1}^{3} \frac{\sigma_{i}}{\gamma_{i}} \widehat{\theta}_{i} \widetilde{\theta}_{i} \leq-\sum_{i=1}^{3} \frac{\widetilde{\theta}_{i}^{2}}{2 \gamma_{i}}+\sum_{i=1}^{3} \frac{\sigma_{i} \theta_{i}^{2}}{2 \gamma_{i}}
\end{aligned}
$$



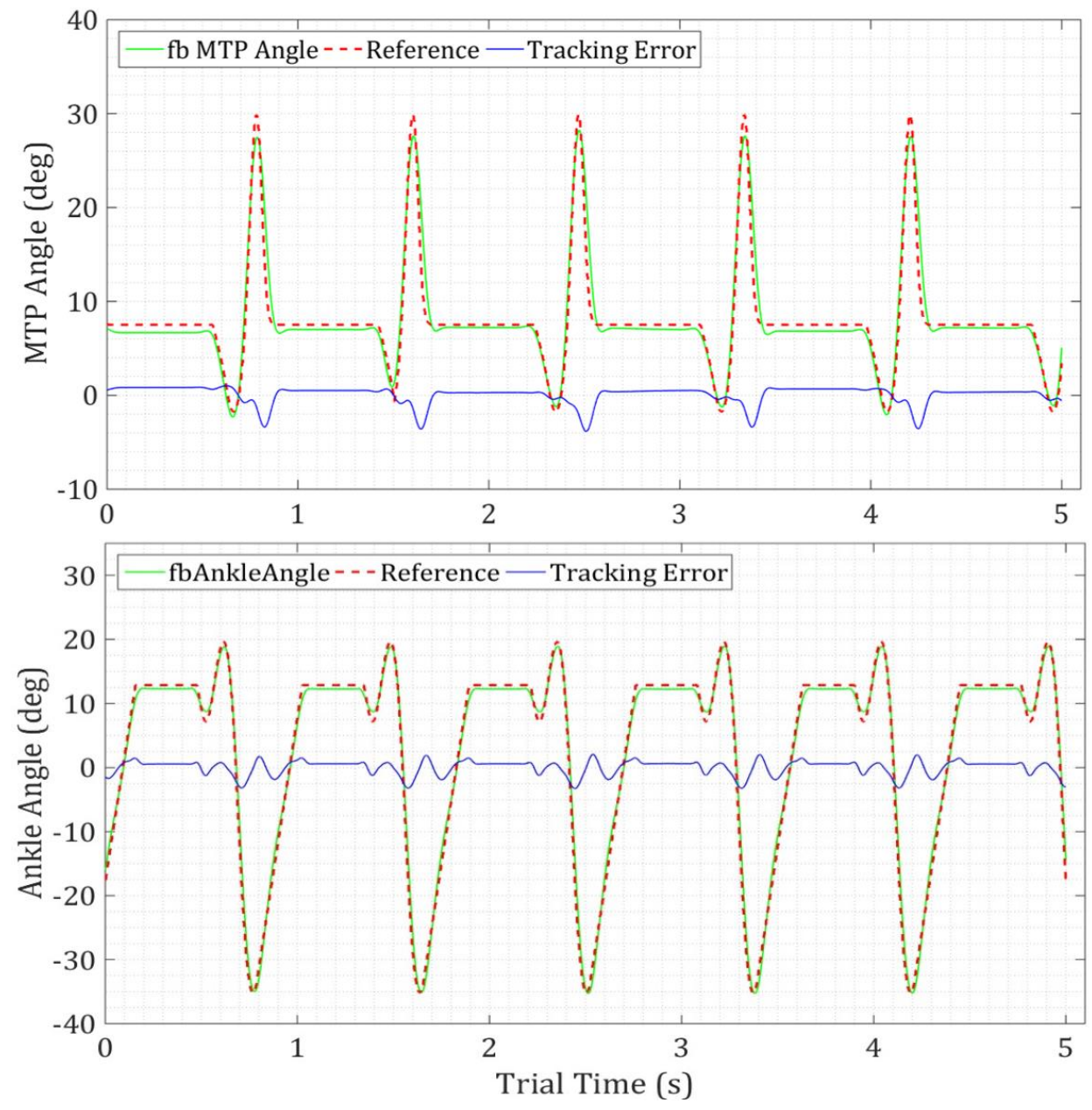

Figure 7: The mimicking performance of the ankle and MTP joints to real reference joint profiles of subject no.7 in heel strike running at $12 \mathrm{~km} / \mathrm{h}[19]$. The RMSEs are $0.9702^{\circ}$ and $1.1437^{\circ}$ for the MTP and ankle joints, respectively. Furthermore, the RMSEs between the references and feedbacks on the specific periods of contact phases are $1.2725^{\circ}$ for the MTP joint and $1.2536^{\circ}$ for the ankle joint. The dash lines plot the human reference profiles of the ankle and MTP joints. The thick continuous lines depict the measured output angles while thin continuous lines represent the tracking errors for the two foot joints.

Hence, inequality Eq. (22) becomes Eq. (27):

$$
\begin{aligned}
\dot{V} & \leq-\lambda z_{1}^{2}-k z_{2}^{2}-\sigma_{\psi} \frac{\alpha \widetilde{\psi}^{2}}{2 \delta}-\sum_{i=1}^{3} \frac{\widetilde{\theta}_{i}^{2}}{2 \gamma_{i}}-\sigma_{b} \frac{\tilde{b}^{2}}{2 \varrho} \\
& -\sigma_{H} \frac{\tilde{H}^{2}}{2 \mu}+\frac{\sigma_{\psi} \alpha \psi^{2}}{2 \delta}+\sum_{i=1}^{3} \frac{\sigma_{i} \theta_{i}^{2}}{2 \gamma_{i}}+\frac{\sigma_{b} b^{2}}{2 \varrho} \\
& +\frac{\sigma_{H} H^{2}}{2 \mu} \leq-v V+\Phi
\end{aligned}
$$

in which $v$ and $\Phi$ are two constant positive quantities as: $v=\min \left(2 \lambda, 2 k, \sigma_{\psi}, s_{i}, s_{b}, s_{H}\right)$ and $\Phi=\sigma_{\psi} \alpha \psi^{2} / 2 \delta+$ $\sum_{i=1}^{3}\left(\sigma_{i} \theta_{i}^{2} / 2 \gamma_{i}\right)+\sigma_{b} b^{2} / 2 \varrho+\sigma_{H} H^{2} / 2 \mu$. From inequality Eq. (27), one can observe that $\forall V \geq V^{*}=\Phi / v$ makes $\dot{V} \leq 0$. This implies that $V$ is a non-increasing function, therefore, all signals in the Lyapunov function $z_{i}, \widetilde{\psi}, \widetilde{\theta}_{i}, \tilde{b}$, and $\tilde{H}$ are bounded. Thus, the estimates $\widehat{\psi}, \widehat{\theta}_{i}, \hat{b}$, and $\hat{H}$ are also bounded.

Combining these with Assumptions (ii) - (iv) and control laws Eq. (15) implies that the control signals $\bar{u}_{c}$ and $u_{c}$ are bounded. Then, Eq. (16) implies that $\dot{\hat{\psi}}, \dot{\hat{H}}, \dot{\hat{\theta}}_{i}$, and $\dot{\hat{b}}$ are bounded. Furthermore, multiply both sides of Eq. (27) with $e^{v t}$ and then integrate two sides of the in- equality, one can obtain Eq. (28):

$$
0 \leq \frac{1}{2} z_{i}^{2} \leq V(t) \leq\left[V\left(t_{0}\right)-\frac{\Phi}{v}\right] e^{-v\left(t-t_{0}\right)}+\frac{\Phi}{v}
$$

in which $V\left(t_{0}\right)$ is obtained at the initial moment $t_{0}$ when the errors $z_{i}$ are zero. Then, one has Eq. (29):

$$
\left|z_{i}\right| \leq \sqrt{2\left[V\left(t_{0}\right)-\frac{\Phi}{v}\right] e^{-v\left(t-t_{0}\right)}+\frac{2 \Phi}{v}}
$$

When $t$ increases, the first term $\left[V\left(t_{0}\right)-\Phi / v\right] e^{-v\left(t-t_{0}\right)} \rightarrow$ 0 . This implies that tracking errors $z_{i}$ converge to a proximity of zero with the bound $\Delta$ as $\left|z_{i}\right| \leq \Delta=\sqrt{2 \Phi / v}$. As the bounds are independent of $t_{0}$, all signals are uniformly ultimately bounded and Theorem 1 holds.

\section{Acknowledgement}

This project was supported by the Institute for Sports Research, Nanyang Technological University, Singapore. 


\section{References}

[1] A.A. Aines. "Mechanical footwear testing machine". Pat. US Patent 2,638,776. 1953.

[2] J. M. Caputo and S. H. Collins. "A universal anklefoot prosthesis emulator for human locomotion experiments". In: Journal of Biomechanical Engineering 136.3 (2014). DOI: 10.1115/1.4026225.

[3] SATRA Technology Centre. SATRA Pedatron Test Machine STM 528. 2010, accessed on 9 July 2017. URL: http : / / satratechnology . com / pedatron . php.

[4] J. T. Cheung et al. "Current methods in computeraided engineering for footwear design". In: Footwear Science 1.1 (2009), pp. 31-46.

[5] K.G. Chrystall, S.P. Monckton, and M. Oleson. "Motion platform and method of use". Pat. US Patent 6,581,437. 2003.

[6] A. Cohen. "Testing footwear soles". Pat. US Patent 4,096,733. 1978.

[7] T. N. Do et al. "Adaptive control for enhancing tracking performances of flexible tendonsheath mechanism in natural orifice transluminal endoscopic surgery (NOTES)". In: Mechatronics 28 (2015), pp. 67-78.

[8] T. N. Do et al. "Nonlinear friction modelling and compensation control of hysteresis phenomena for a pair of tendon-sheath actuated surgical robots". In: Mechanical Systems and Signal Processing 6061.0 (2015), pp. 770-784.

[9] T. N. Do et al. "Position Control of Asymmetric Nonlinearities for a Cable-Conduit Mechanism". In: IEEE Transactions on Automation Science and Engineering 14.3 (2017), pp. 1515-1523.

[10] Albert Einstein. "Zur Elektrodynamik bewegter Körper. (German) [On the electrodynamics of moving bodies]". In: Annalen der Physik 322.10 (1905), pp. 891-921. DOI: http://dx.doi.org/10.1002/ andp. 19053221004.

[11] J. Heidenfelder, T. Sterzing, and T. L. Milani. "Biomechanical wear testing of running shoes". In: Footwear Science 1.sup1 (2009), pp. 16-17.

[12] E. M. Hennig. "Eighteen years of running shoe testing in Germany a series of biomechanical studies". In: Footwear Science 3.2 (2011), pp. 71-81.

[13] E. M. Hennig and S. Fischer. "Changes in footwear properties after 50, 200, 500 and $1000 \mathrm{~km}$ of running". In: 16th Biannual Conference of the Canadian Society for Biomechanics. Vol. 87. Kingston, Ontario, Canada: Queens University, 2010.

[14] E.B. Hovey and D.O. White. "Testers for shoes". Pat. US Patent 3,608,372. 1971.

[15] F. Ikhouane and J. Rodellar. Systems with Hysteresis: Analysis, Identification and Control using the Bouc-Wen Model. Systems with Hysteresis: Analysis, Identification and Control using the Bouc-Wen Model. John Wiley \& Sons, 2007, pp. 1-202.

[16] P. A. Ioannou and J. Sun. Robust adaptive control. Courier Corporation, 2012.
[17] T. Lenzi et al. "NEUROExos: A variable impedance powered elbow exoskeleton". In: Robotics and $A u$ tomation (ICRA), 2011 IEEE International Conference on. IEEE. 2011, pp. 1419-1426.

[18] C. R. Miguel. A 3D human foot. 2014, accessed on 3 January 2016. URL: https : / / grabcad . com / library/human-foot-1.

[19] T. L. Nguyen. "Development of a Robotic Running Foot for Footwear Testing". PhD thesis. Nanyang Technological University, Singapore, 2017.

[20] T. L. Nguyen, S. J. Allen, and S. J. Phee. "Exploiting 3D printing technology to develop robotic running foot for footwear testing". In: Virtual and Physical Prototyping 8.4 (2013), pp. 259-269.

[21] T. L. Nguyen, S. J. Allen, and S. J. Phee. "Motion Control in Torque Mode for a Robotic Foot for Footwear Testing". In: 2nd International Conference in Sports Science Technology (ICSST). 2016, 240245.

[22] T. L. Nguyen et al. "Modelling, design, and control of a robotic running for footwear testing with flexible actuator". In: 1st International Conference in Sports Science Technology (ICSST). 2014, 505-514.

[23] T. F. Novacheck. "The biomechanics of running". In: Gait \&3 Posture 7.1 (1998), pp. 77-95.

[24] E.G. Paquette et al. "Footwear testing apparatus and method". Pat. US Patent 4,432,223. 1984.

[25] F.J. Pitman and R.J. Chouinard. "Wear tester for shoes". Pat. US Patent 4,327,572. 1982.

[26] P. O. Riley et al. "A kinematics and kinetic comparison of overground and treadmill running". In: Med Sci Sports Exerc 40.6 (2008), pp. 1093-100.

[27] J. A. Ronkainen et al. "Application of an industrial robot in the sports domain: Simulating the ground contact phase of running". In: Proceedings of the Institution of Mechanical Engineers, Part P: Journal of Sports Engineering and Technology 224.4 (2010), pp. 259-269.

[28] S.E. Taub. "Wear-testing method". Pat. US Patent 3,516,281. 1970.

[29] R. Therrio et al. "Footwear testing machine". Pat. US Patent App. 11/419,458. 2007.

[30] J. F. Veneman. "A Series Elastic- and BowdenCable-Based Actuation System for Use as Torque Actuator in Exoskeleton-Type Robots". In: The International Journal of Robotics Research 25.3 (2006), pp. 261-281.

[31] F. Zhang et al. "Design and development of a hand exoskeleton for rehabilitation of hand injuries". In: Mechanism and Machine Theory 73.0 (2014), pp. 103-116. 